\title{
A study of the double hadron neutrinoproduction on nuclei
}

\author{
SKAT Collaboration \\ N.M. Agababyan ${ }^{1}$, V.V. Ammosov², M. Atayan ${ }^{3}$, \\ L. Grigoryan ${ }^{3}$, N. Grigoryan ${ }^{3}$, H. Gulkanyan ${ }^{3}$, \\ A.A. Ivanilov ${ }^{2}$, Zh. Karamyan ${ }^{3}$, V.A. Korotkov ${ }^{2}$ \\ 1 Joint Institute for Nuclear Research, Dubna, Russia \\ ${ }^{2}$ Institute for High Energy Physics, Protvino, Russia \\ ${ }^{3}$ Yerevan Physics Institute, Armenia
}




\begin{abstract}
The nuclear medium influence on the dihadron neutrinoproduction is investigated for the first time, using the data obtained with SKAT bubble chamber. An indication is obtained that the nuclear attenuation of the dihadron is more expressed for kinematically closest hadron pairs. The experimental data on the dihadron attenuation and on the ratio of the dihadron to single-hadron yields are compared with predictions of the two-scale string fragmentation model.
\end{abstract}




\section{Introduction}

The space-time structure of the quark string fragmentation in leptoproduction reactions imposes certain correlations between two-hadron formation processes. A unique information on the latter can be inferred from experiments on nuclear targets $([1,42,43,4$, 5] and references therein). The first measurements of the dihadron leptoproduction, performed recently [6] using a $27.6 \mathrm{GeV}$ positron beam with deuterium, nitrogen, krypton and xenon targets, showed that the available theoretical models do not satisfactorily describe the experimental data, especially on heavier targets.

The aim of the present work is to obtain the first experimental data on the dihadron neutrinoproduction at comparatively lower energies, at which the nuclear attenuation effects are expected to be more prominent. To this end, the data from the SKAT bubble chamber [7] were used. In Section 2, the experimental procedure is described. Section 3 presents the data on the dihadron attenuation versus its kinematical variables. In Section 4, the data on the dihadron attenuation are compared with those for the single hadron and with theoretical predictions. The results are summarized in Section 5.

\section{Experimental procedure}

The experiment was performed with SKAT bubble chamber, exposed to a wideband neutrino beam obtained with a $70 \mathrm{GeV}$ primary protons from the Serpukhov accelerator. The chamber was filled with a propane-freon mixture containing 87 vol\% propane $\left(C_{3} H_{8}\right)$ and 13 vol\% freon $\left(\mathrm{CF}_{3} \mathrm{Br}\right)$ with the percentage of nuclei $\mathrm{H}: \mathrm{C}: \mathrm{F}: \mathrm{Br}=67.9: 26.8: 4.0: 1.3 \%$. A $20 \mathrm{kG}$ uniform magnetic field was provided within the operating chamber volume.

Charged current interactions containing a negative muon with momentum $p_{\mu}>0.5 \mathrm{GeV} / \mathrm{c}$ were selected. Other negatively charged particles were considered to be $\pi^{-}$mesons. Protons with momentum below $0.6 \mathrm{GeV} / c$ and a fraction of protons with momentum $0.6-0.85 \mathrm{GeV} / c$ were identified by their stopping in the chamber. Non-identified positively charged particles were considered to be $\pi^{+}$mesons. Events in which errors in measuring the momenta of all charged secondaries and photons were less than $27 \%$ and $100 \%$, respectively, were selected. Each event is given a weight which corrects for the fraction of events excluded due to improperly reconstruction. More details concerning the experimental procedure, in particular, the reconstruction of the neutrino energy $E_{\nu}$ can be found in our previous publications [8, 9]. The events with $3<E_{\nu}<30 \mathrm{GeV}$ were accepted, provided that the reconstructed mass $W$ of the hadronic system exceeds $2 \mathrm{GeV}$ and $y=\nu / E_{\nu}<0.95, \nu$ being the energy transferred to the hadronic system. No restriction was imposed on the transfer momentum squared $Q^{2}$. The number of accepted events was 3440 (4819 weighted events). The mean values of the kinematical variables were $\left\langle E_{\nu}\right\rangle=10.0 \mathrm{GeV},\langle W\rangle=2.9 \mathrm{GeV},\left\langle W^{2}\right\rangle=9.1 \mathrm{GeV}^{2}$, $\left.<Q^{2}\right\rangle=2.7(\mathrm{GeV} / \mathrm{c})^{2}$ and $\langle\nu\rangle=5.8 \mathrm{GeV}$.

Further, the whole event sample was subdivided, using several topological and kinematical criteria [9, 10], into three subsamples: the 'cascade' subsample $B_{S}$ with a sign of intranuclear secondary interaction, the 'quasiproton' $\left(B_{p}\right)$ and 'quasineutron' $\left(B_{n}\right)$ subsamples. About $40 \%$ of subsample $B_{p}$ is contributed by interactions with free hydrogen. Weighting the 'quasiproton' events with a factor of 0.6, one can compose a 'pure' nuclear subsample $B_{A}=B_{S}+B_{n}+0.6 B_{p}$ and a 'quasinucleon' subsample $B_{N}=B_{n}+0.6 B_{p}$. It has been verified [9, 10, 11], that the multiplicity and spectral characteristics of secondary particles in the $B_{p}\left(B_{N}\right)$ subsample are in satisfactory agreement with those measured with a pure 
proton (deuteron) target. The effective atomic weight corresponding to the subsample $B_{A}$ is estimated [12] to be approximately equal to $A_{\text {eff }}=21 \pm 2$, when taking into account the probability of secondary intranuclear interactions in the composite target.

\section{The nuclear attenuation versus dihadron kinematical variables}

Below we will consider hadrons produced in the forward hemisphere in the hadronic c.m.s. (i.e. in the region of $x_{F}>0, x_{F}$ being the Feynman variable), because in this region the nuclear attenuation effects for hadrons dominate over intranuclear cascading effects which lead to an enhancement of their yield, observable in the region of $x_{F}<0$ (see e.g. [9, 11]). In this section, we present the data which demonstrate the nuclear medium influence on the dihadron yield in dependence on kinematical variables describing the double-hadron system. For a given variable $v$, this influence is characterized by the ratio $R_{2}(v)$ of the dihadron differential yields $\left\langle n_{2}(v)>_{A}\right.$ and $\left\langle n_{2}(v)\right\rangle_{N}$ in the nuclear and 'quasinucleon' subsamples, $R_{2}(v)=<n_{2}(v)>_{A} /<n_{2}(v)>_{N}$.

Figs. 1-3 demonstrate the dependence of $R_{2}$ on the relative kinematical variables of two hadrons: $\Delta x_{F}=\left|x_{F 1}-x_{F 2}\right| ; \Delta z=\left|z_{1}-z_{2}\right|, z_{1}$ and $z_{2}$ being the fractions of the quark energy $\nu$ carried by hadrons; $\Delta y^{*}=\left|y_{1}-y_{2}\right|, y_{1}$ and $y_{2}$ being the rapidities in the hadronic c.m.s.;

$\eta_{\text {rel }}=-\ln \left[\tan \left(\vartheta_{\text {rel }} / 2\right)\right], \vartheta_{\text {rel }}$ being the angle between two hadrons; $Q_{i n v}=\sqrt{-\left(p_{1}-p_{2}\right)^{2}}, p_{1}$ and $p_{2}$ being the four-momenta of hadrons; $q_{t}=\left|\overrightarrow{p_{t 1}}-\overrightarrow{p_{t 2}}\right|, \overrightarrow{p_{t 1}}$ and $\overrightarrow{p_{t 2}}$ being the transverse momenta of hadrons relative to the current quark direction. The data are presented separately for the like-sign $\left(\pi^{+} \pi^{+}\right)$, unlike-sign $\left(\pi^{+} \pi^{-}\right)$and charged $(\pi \pi)$ hadron pairs.

The most interesting feature of the data of Figs. 1-3 is that the attenuation of the unlikesign pair produced in the quark fragmentation region (at $x_{F}>0.15$ ) tends to strengthen with decreasing relative kinematical variables (the middle panels of figures); the said also concerns the relative angle $\vartheta_{r e l}$ (which decreases with increasing $\eta_{\text {rel }}$ ). This tend, as it can be seen from figures, is not caused by the $\rho^{0}$ meson production. The said feature is less expressed (or even absent) for the like-sign pairs (the top panels of Figs. 1-3). Note also, that no definite dependence of $R_{2}$ on the relative kinematical variables is observed at a less strict cut on $x_{F}\left(x_{F}>0\right)$.

Figs. 4 and 5 show the ratio $R_{2}$ versus the collective kinematical variables of the dihadron: $x_{F}^{\text {pair }}, z^{\text {pair }}$, the rapidity $y^{\text {pair }}$ and the transverse momentum $p_{t}^{\text {pair }}$. For the case of $x_{F}>0.15$, no significant dependence on collective variables is observed, except a possible rise of $R_{2}$ with increasing $z^{\text {pair }}$ (the right panel of Fig. 4). For the case of the cut $x_{F}>0$, the value $R_{2}$ is consistent with 1 at the smallest value of $x_{F}^{\text {pair }}, z^{\text {pair }}$, and $y^{\text {pair }}$, while at larger values of the latters a significant attenuation $\left(R_{2}<1\right)$ is observed. An indication is obtained, that the attenuation of the like-sign pair at $x_{F}>0$ weakens with increasing $p_{t}^{\text {pair }}$ (Fig. 5 , the right top panel).

\section{The dihadron versus single-hadron attenuation}

The correlation between the two-hadron attenuation as a function of certain kinematical variable $v$ can be characterized by the double ratio 


$$
r_{21}\left(v_{1}, v_{2}\right)=\frac{R_{2}\left(v_{1}, v_{2}\right)}{R_{1}\left(v_{1}\right)}
$$

where the ratio $R_{1}(v)=<n_{1}(v)>_{A} /<n_{1}(v)>_{N}$ is the single-hadron attenuation factor. It should be stressed here, that even at the absence of dinamical correlations between attenuations of two hadrons, the paths they pass in the nuclear medium (and hence their absorption probabilities) are always 'geometrically' correlated, resulting in a certain dependence of $r_{21}\left(v_{1}, v_{2}\right)$ on $v_{2}$ (at fixed $v_{1}$ ). If the nuclear attenuation is dominated by the absorption of the (pre)hadronic states, then the double ratio $r_{21}\left(v_{1}, v_{2}\right)$ is always obviously smaller than 1 . Below we will consider the dependence of $r_{21}\left(z_{1}, z_{2}\right)$ on variables $z_{1}$ and $z_{2}$ of two hadrons.

Before presenting the data on the double ratio, it will be useful to consider separately the single-hadron and dihadron attenuation factors $R_{1}\left(z_{1}\right)$ and $R_{2}\left(z_{1}, z_{2}\right)$. The latters are plotted in Figs. 6 and 7 for charged hadrons with $x_{F}>0$ and $x_{F}>0.1$, respectively. The values of $R_{1}\left(z_{1}\right)$ are quoted numerically and indicated, for visual comparison with $R_{2}\left(z_{1}, z_{2}\right)$, by dashed lines. As it is seen, in general $R_{2}\left(z_{1}, z_{2}\right)<R_{1}\left(z_{1}\right)$ due to the additional attenuation of the yield of the accompanying hadron with $z=z_{2}$.

As it was already mentioned in the previous section, the attenuation is more expressed for kinematically close pairs, in particular, at $z_{1}, z_{2}<0.12$ and $0.24<z_{1}, z_{2}<0.37$ (Fig. 6) and $0.12<z_{1}, z_{2}<0.24$ and $0.24<z_{1}, z_{2}<0.34$ (Fig. 7). Similar observations can be done from Fig. 8 and 9, where the double ratio $r_{21}\left(z_{1}, z_{2}\right)$ is plotted as a function of $z_{1}$ and $z_{2}$ for charged hadrons with $x_{F}>0$ and $x_{F}>0.1$, respectively.

The double ratio $r_{21}\left(z_{1}, z_{2}\right)$ was recently measured in electronuclear interactions [4], at $z_{1}=z_{t r}>0.5$ for the leading trigger particle and $z_{2}=z_{s u b}$ for the subleading particle, being the fastest one among particles accompanying the leading particle. Fig. 10 presents the data of [4] on the nitrogen target $(A=14)$ for charged pion combinations (the top panel) and, in the bottom panel, for all pion combinations (including $\pi^{0}$ mesons), except $\pi^{+} \pi^{-}$, in order to exclude the contribution from the $\rho^{0}$ decay. In the same figure we also plot our data for charged pion combinations (the top panel), for combinations including also $\pi^{0}$ mesons (the middle panel), and the same but without $\pi \pi$ combinations from the $\rho$ mass region. As it is seen, our data are mainly compatible with electroproduction data, exhibiting, however, an enhancement at the low- $z_{2}$ region, probably due to the more pronounced intranuclear cascading effects in a slightly heavier composite target $\left(A_{\text {eff }} \approx 21\right)$ used in our experiment. We undertook an attempt to describe our data on $R_{1}(z), R_{2}\left(z_{1}, z_{2}\right)$ and the double ratio $r_{21}\left(z_{1}, z_{2}\right)$ in the framework of the Two-Scale Model (TSM) [13] (see also [5, 14]) which incorporates the space-time pattern of the quark string fragmentation followed from the Lund model [1, 15]. The TSM contains four free parameters: the quark string tension $\kappa$ and the following string-nucleon interaction cross sections, namely, $\sigma_{q}$ for the initial string stretched between the struck quark and the target nucleon remnant, $\sigma_{s}$ for the intermediate string stretched between the struck quark and a created antiquark (which becomes a valence one for the hadron being looked at) and $\sigma_{h}$ for the formed colorless system with quantum numbers and valence content of the final hadron.

First of all we checked the compatibility of our data on the single hadron attenuation factor with the TSM. A satisfactory description of the data on $R_{1}(z)$ for hadrons with $x_{F}>0.1$ (quoted in Fig. 7), as well as for hadrons with $x_{F}>0.1$ and $z>0.5$ (quoted in Fig. 6, the left bottom panel) can be reached at the following set of the model parameters: $\kappa=0.8$ $\mathrm{GeV} / \mathrm{fm}, \sigma_{q}=0, \sigma_{s}=10 \mathrm{mb}$ and $\sigma_{h}=20 \mathrm{mb}$ (note, that this set somewhat differs from those estimated in [13] and [14]). The TSM predictions for $R_{2}\left(z_{1}, z_{2}\right)$ and $r_{21}\left(z_{1}, z_{2}\right)$, obtained 
with these parameters, are plotted in Figs. 7, 9 and 10. As it is seen, the model predictions are, in general, compatible with experimental data. The model, however, does not describe the reduced yield of the pair of low- $z$ hadrons with $0.12<z_{1}, z_{2}<0.24$ (Figs. 7 and 9, the left top panel). A similar inconsistency is also seen for $R_{2}(\Delta z)$ at low values of variable $\Delta z=\left|z_{1}-z_{2}\right|$ for charged hadrons with $x_{F}>0.15$ (Fig. 1, the right bottom panel). On the other hand, the model underestimates the value of $R_{2}\left(z^{\text {pair }}\right)$ at largest values on the collective variable $z^{\text {pair }}=z_{1}+z_{2}$ for charged hadrons with $x_{F}>0.1$ (Fig. 4, the right bottom panel). Note also, that the data on the double ratio $r_{21}\left(z_{1}>0.5, z_{2}\right)$ at $z_{2}<0.24$ (Fig. 10, the bottom panel) significantly exceed the model predictions, probably due to the fact that the latter do not incorporate the intranuclear cascading processes which could enhance the yield of low- $z$ hadrons, as well as due to a possible contamination of the data by non-identified protons.

\section{Summary}

For the first time for neutrinonuclear interactions, the nuclear medium influence on the dihadron production is investigated, at an effective atomic weight $A_{\text {eff }} \approx 21$ of a composite target.

The dihadron attenuation factor, $R_{2}$, is measured versus various kinematical veriables of the dihadron. An indication is obtained, that the nuclear attenuation strengthens with decreasing relative variables of two hadrons, reaching about $R_{2} \approx 0.7$ for kinematically closest pairs. It is shown, that the experimental data on the attenuation factor $R_{2}\left(z_{1}, z_{2}\right)$ and the double ratio $r_{21}\left(z_{1}, z_{2}\right)=R_{2}\left(z_{1}, z_{2}\right) / R_{1}\left(z_{1}\right)$ are compatible with predictions of the two-scale string fragmentation model, except for the case of pairs of low- $z$ hadrons with $0.12<z_{1}, z_{2}<0.24$ and pairs of a low- $z(z<0.24)$ subleading and a high- $z(z>0.5)$ leading hadrons.

Acknowledgement. The authors from YerPhI acknowledge the supporting grants of Calouste Gulbenkian Foundation and Swiss Fonds "Kidagan". The activity of two of the authors (L.G. and H.G.) is supported by Cooperation Agreement between DESY and YerPhI signed on December 6, 2002.

\section{References}

[1] J.Czyžewski, Phys. Rev. C 43, 2426 (1991)

[2] B.Z.Kopeliovich et al., Nucl. Phys. A 740, 211 (2004)

[3] A.Majumder, nucl-th/0503019

[4] T.Falter, K.Gallmeister, U.Mozel, nucl-th/0509041

[5] N.Akopov, L.Grigoryan, Z.Akopov, hep-ph/0605128

[6] A.Airapetian et al. (HERMES Coll.), Phys. Rev. Lett. 96, 162301 (2006)

[7] V.V.Ammosov et al. Fiz. Elem. Chastits At. Yadra 23, 648, 1992 [Sov. J. Part. Nucl. 23, 283, (1992)]

[8] N.M.Agababyan et al. (SKAT Coll.), YerPhI Preprint N 1535 (Yerevan, 1999)

[9] N.M.Agababyan et al. (SKAT Coll.), Yad. Fiz. 66, 1350 (2003) [Phys. of At. Nucl. 66, 1310 (2003)]

[10] N.M.Agababyan et al. (SKAT Coll.),YerPhI Preprint N 1578 (Yerevan, 2002)

[11] N.M.Agababyan et al. (SKAT Coll.), Yad. Fiz. 68, 1241 (2005) [Phys. of At. Nucl. 68, $1160(2005)]$ 
[12] N.M.Agababyan et al. (SKAT Coll.), hep-ex/0504024; YerPhI Preprint N 1597 (Yerevan, 2005);

[13] J.Ashman et al. Z. Phys. C 52, 1 (1991)

[14] N.Akopov, L.Grigoryan, Z.Akopov, Eur. Phys. J. C 44, 219 (2005)

[15] A.Bialas, M.Gyulassy, Nucl. Phys. B 291, 793 (1987)

[16] A.Airapetian et al. (HERMES Coll.), Eur. Phys. J. C 20, 479 (2001) 

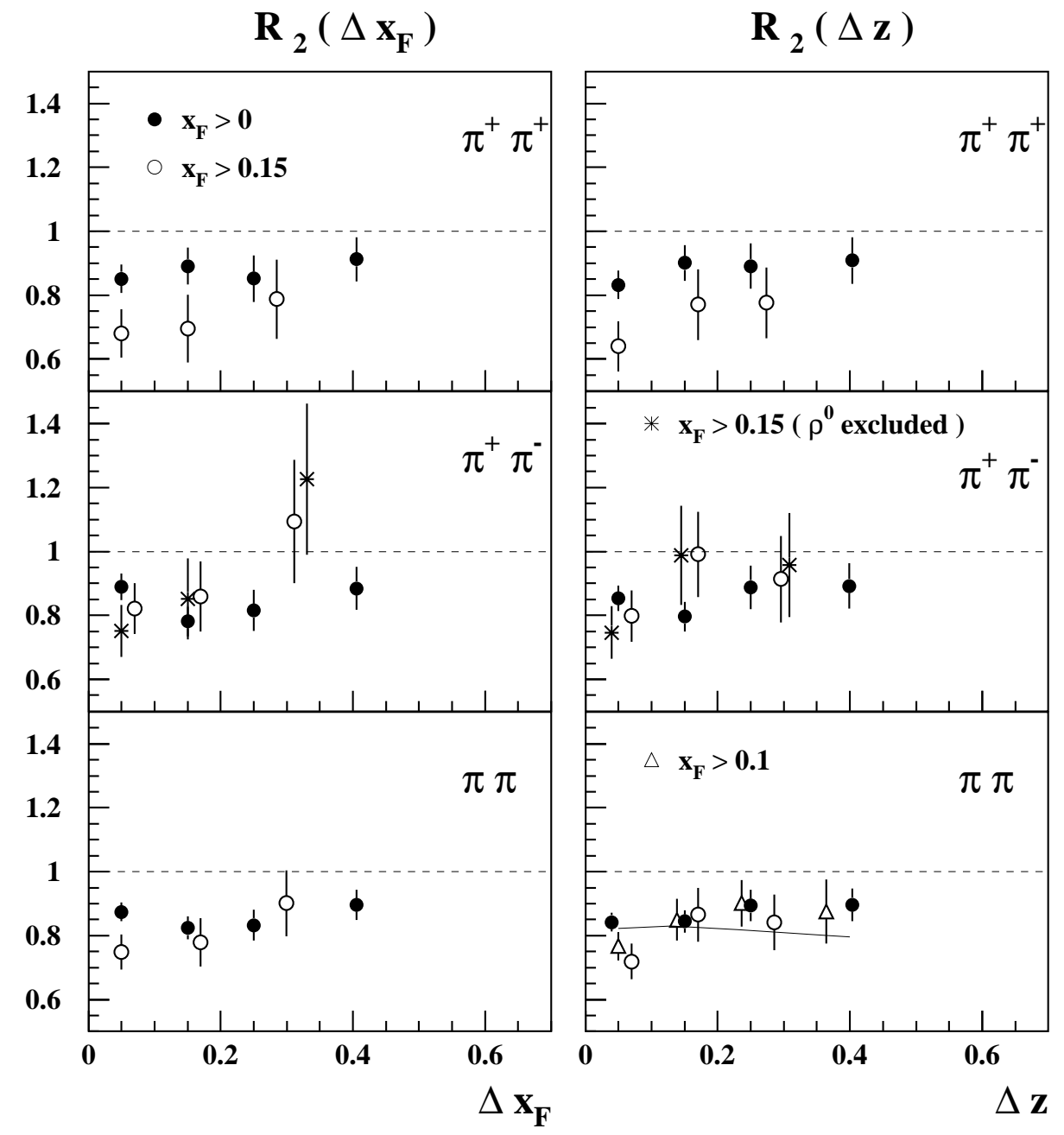

Figure 1: The dependence of the dihadron attenuation factor $R_{2}$ on $\Delta x_{F}$ (left panel) and $\Delta z$ (right panel). The curve is the model prediction (see text). 

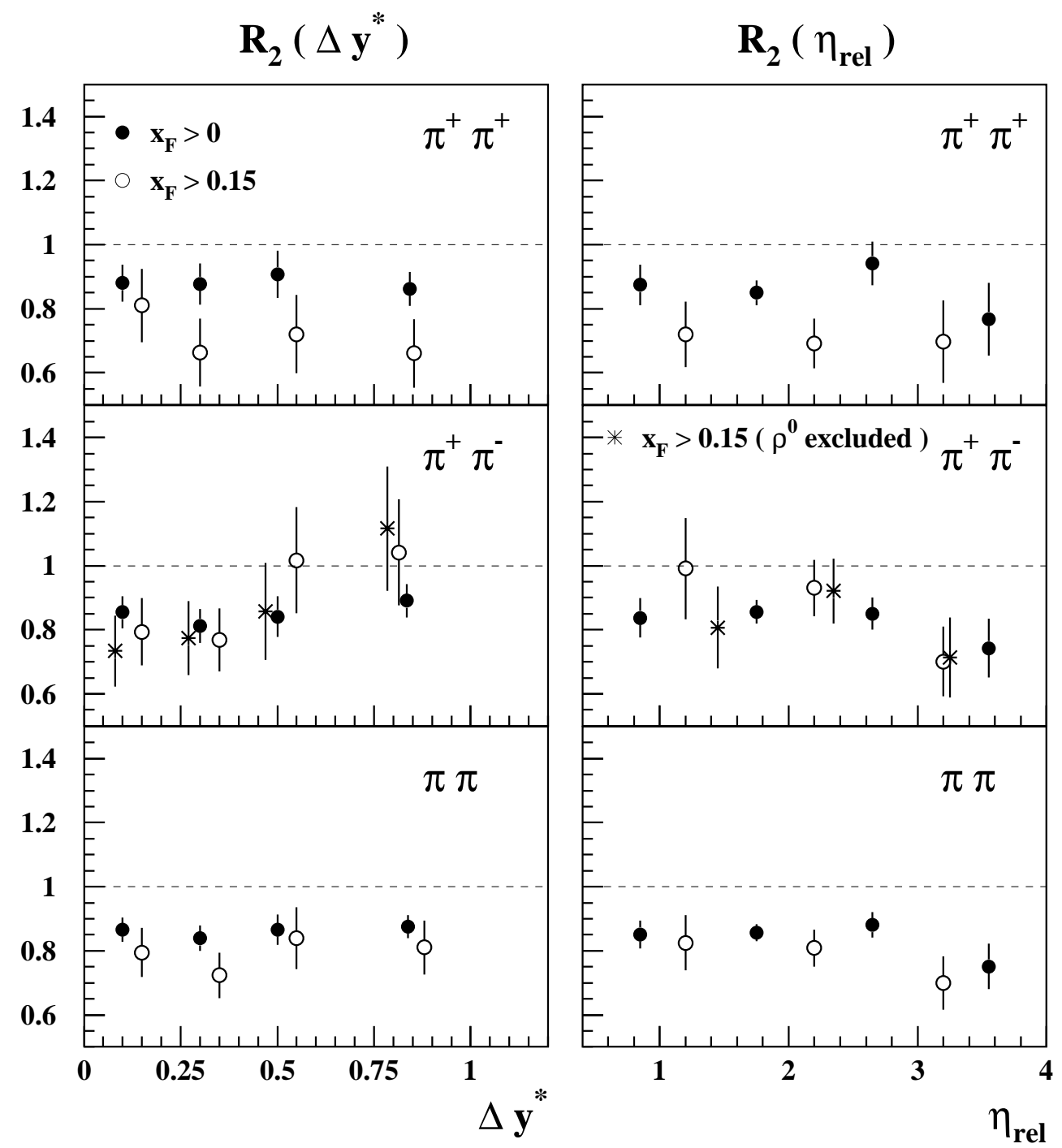

Figure 2: The dependence of the dihadron attenuation factor $R_{2}$ on $\Delta y^{*}$ (left panel) and $\eta_{\text {rel }}$ (right panel). 

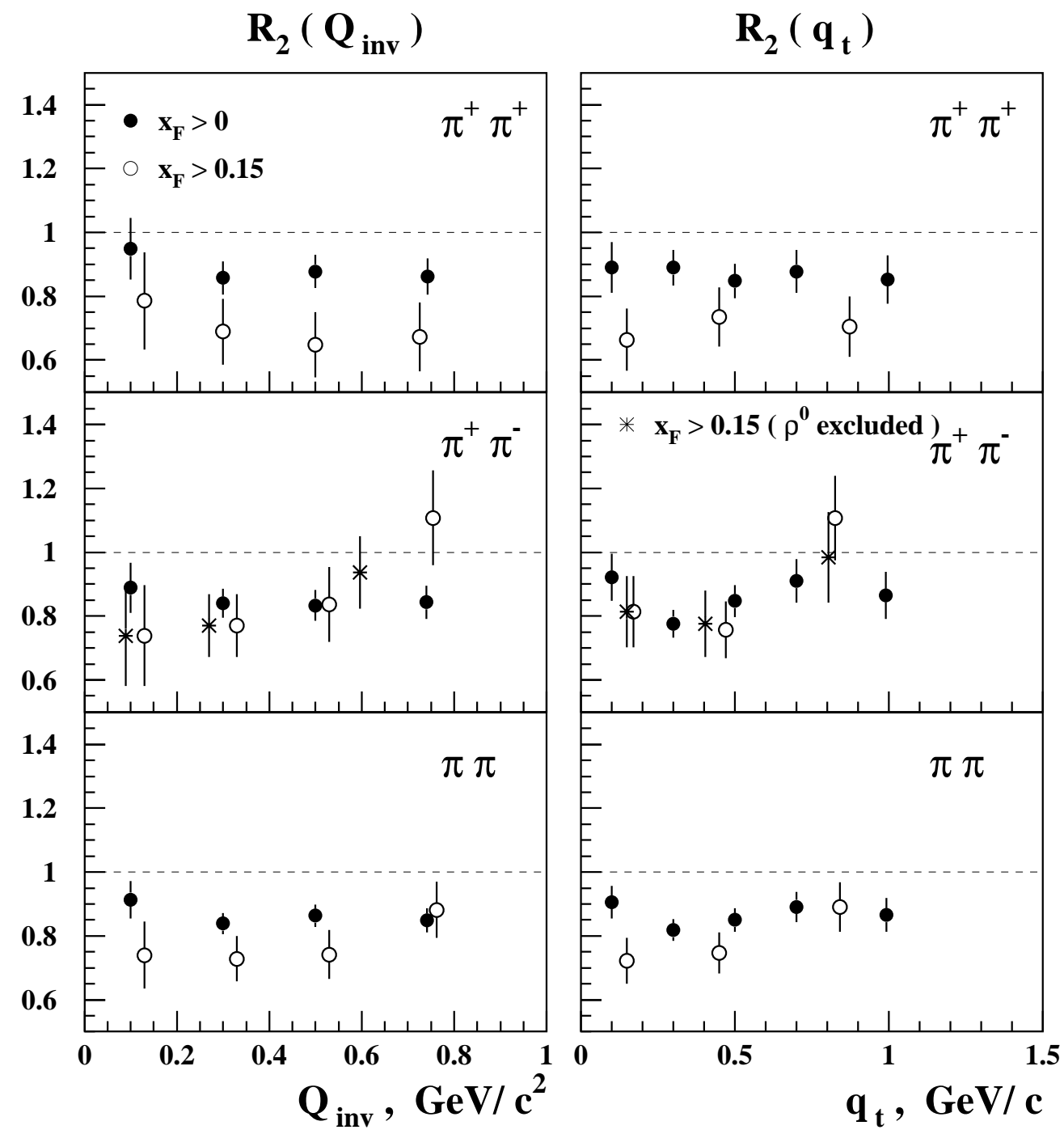

Figure 3: The dependence of the dihadron attenuation factor $R_{2}$ on $Q_{i n v}$ (left panel) and $q_{t}$ (right panel). 

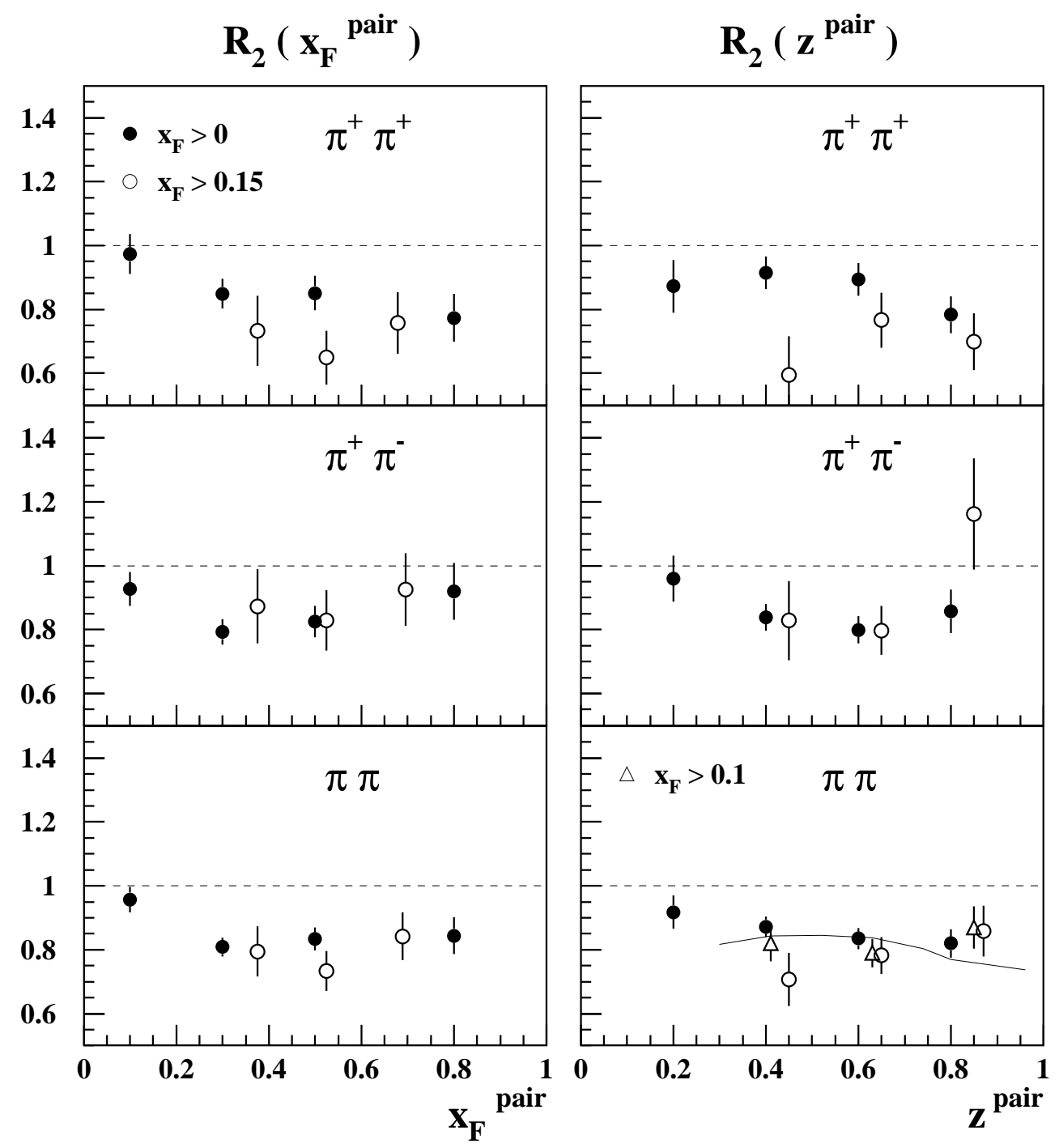

Figure 4: The dependence of the dihadron attenuation factor $R_{2}$ on $x_{F}^{\text {pair }}$ (left panel) and $z^{\text {pair }}$ (right panel). The curve is the model prediction (see text). 


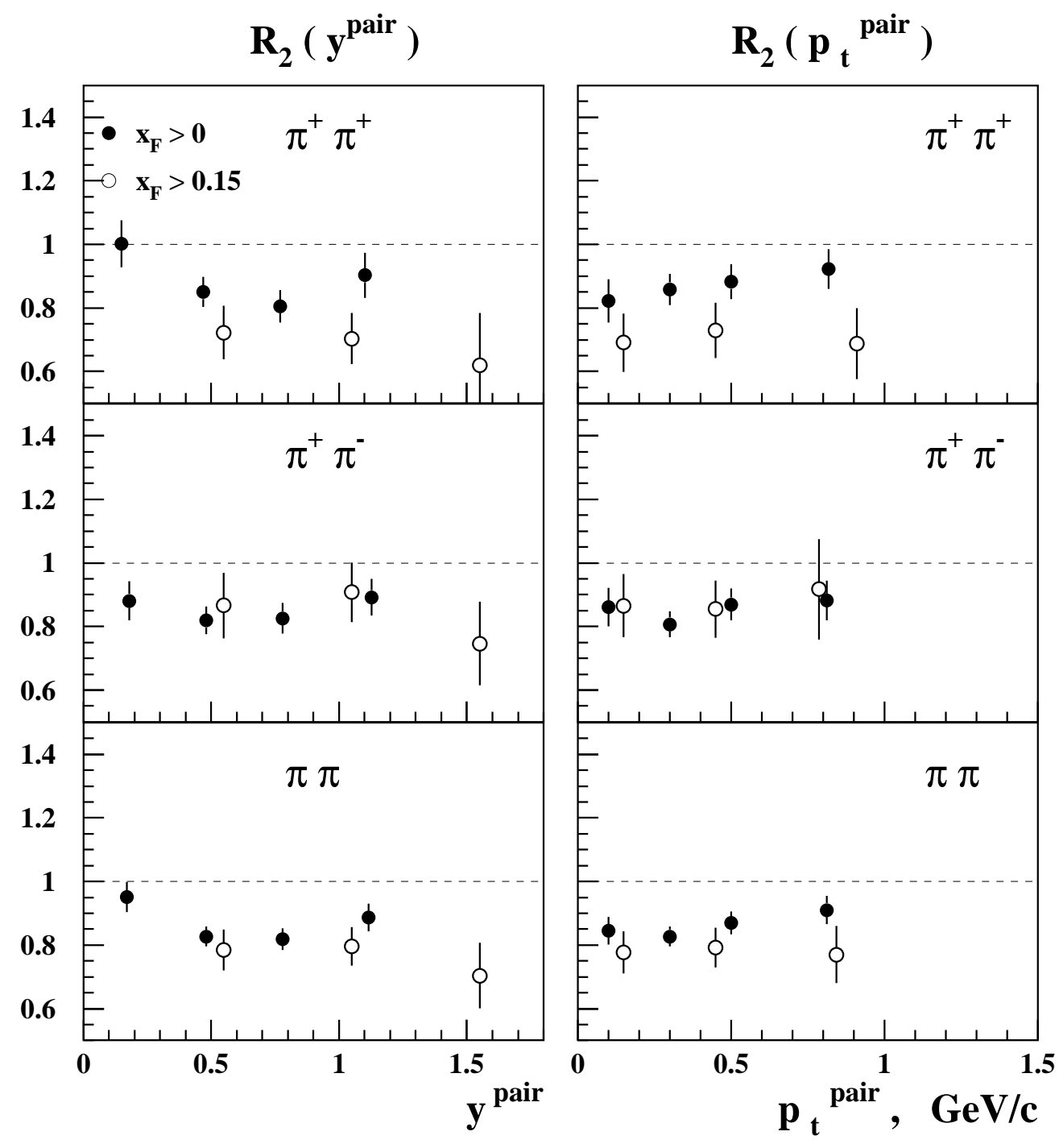

Figure 5: The dependence of the dihadron attenuation factor $R_{2}$ on $y^{\text {pair }}$ (left panel) and $p_{t}^{\text {pair }}$ (right panel). 


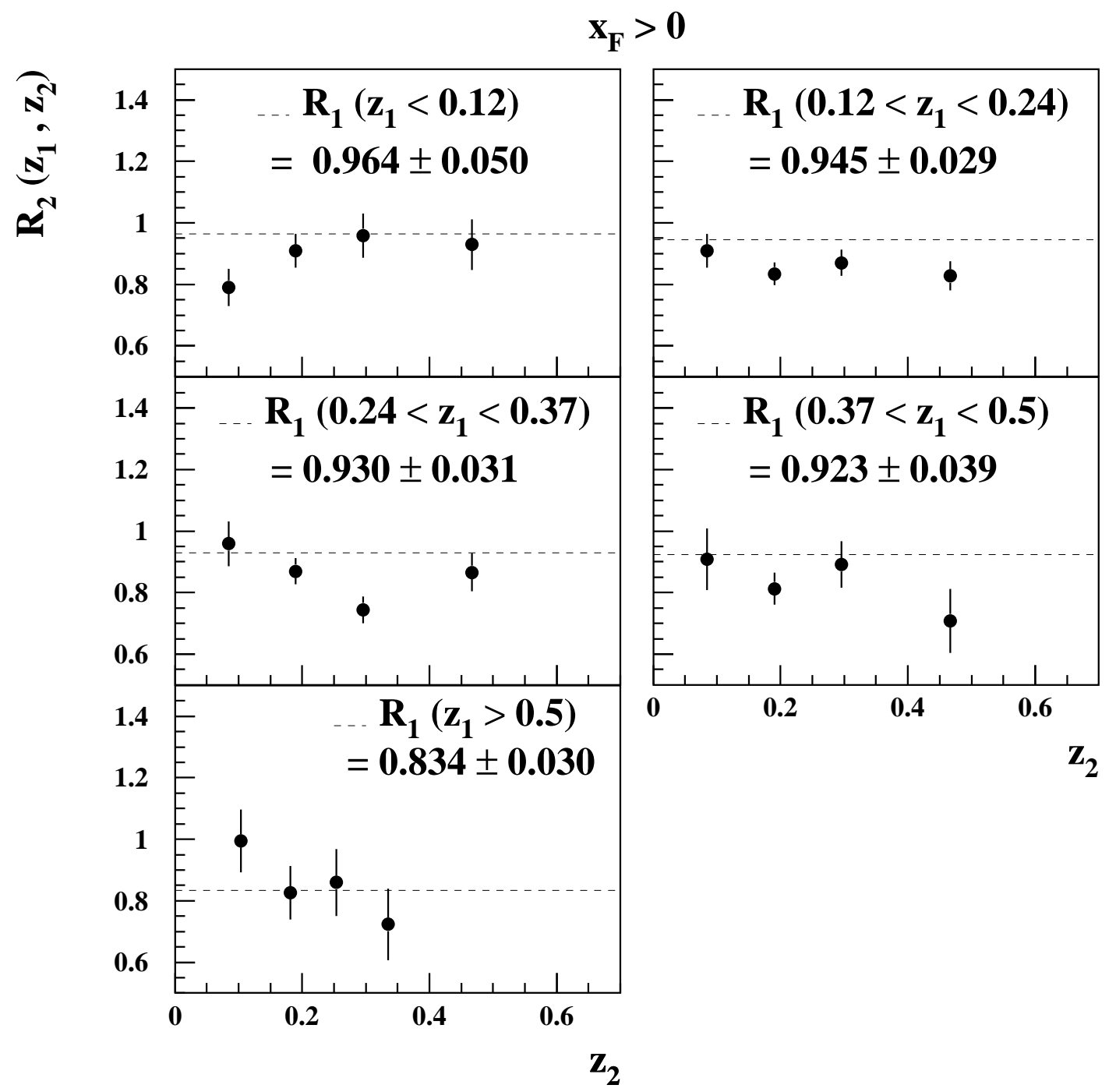

Figure 6: The dependence of the dihadron attenuation factor $R_{2}\left(z_{1}, z_{2}\right)$ as a function of $z_{1}$ (for 'trigger' particle) and $z_{2}$ (for accompanying particle). The numerical values and the dashed lines in figures concern the single-particle attenuation factor $R_{1}\left(z_{1}\right)$. The cut $x_{F}>0$ was applied. 


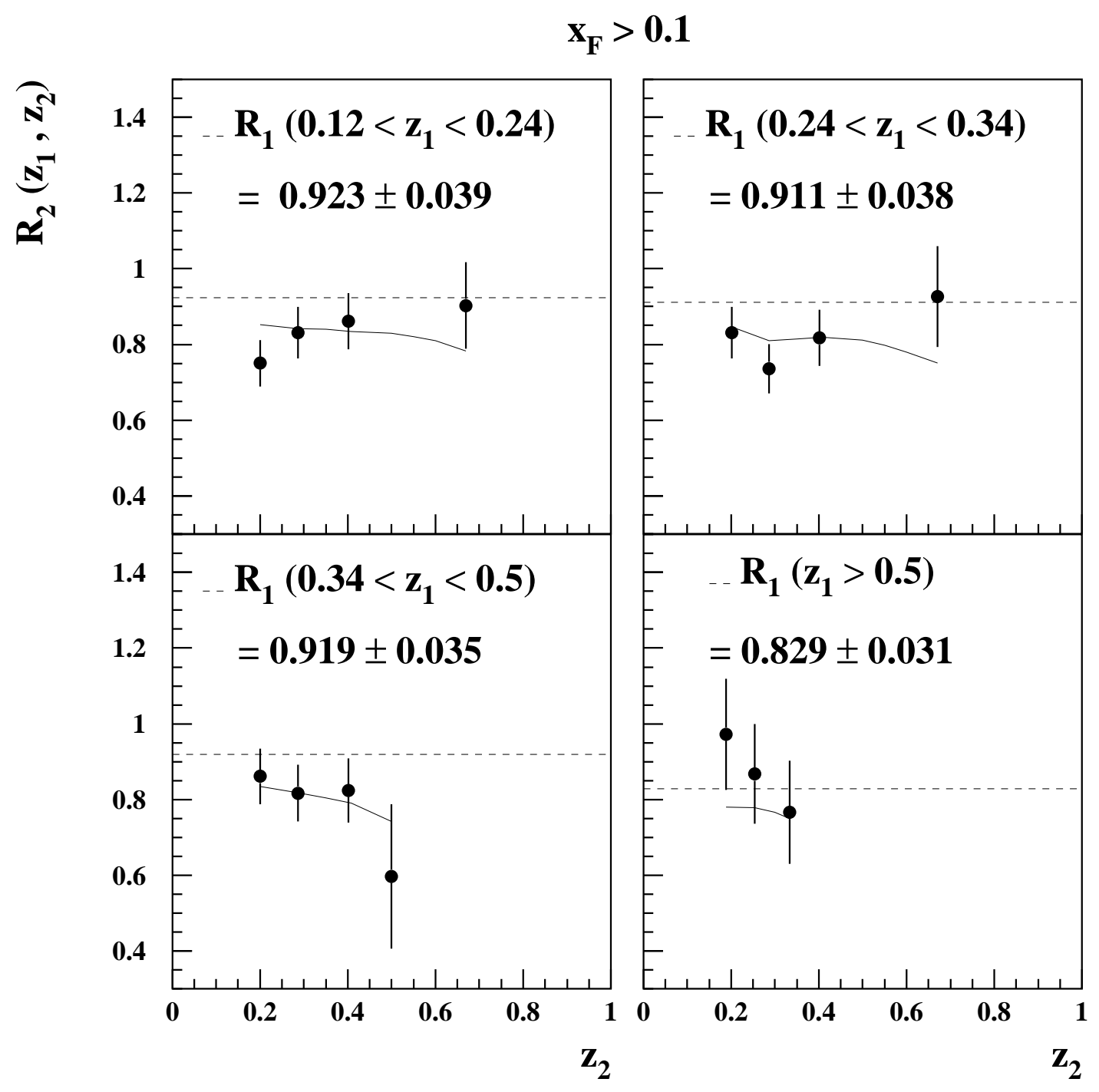

Figure 7: The same as Figure 6, but the cut $x_{F}>0.1$ is applied. The curves are predictions of the two-scale string fragmentation model. 


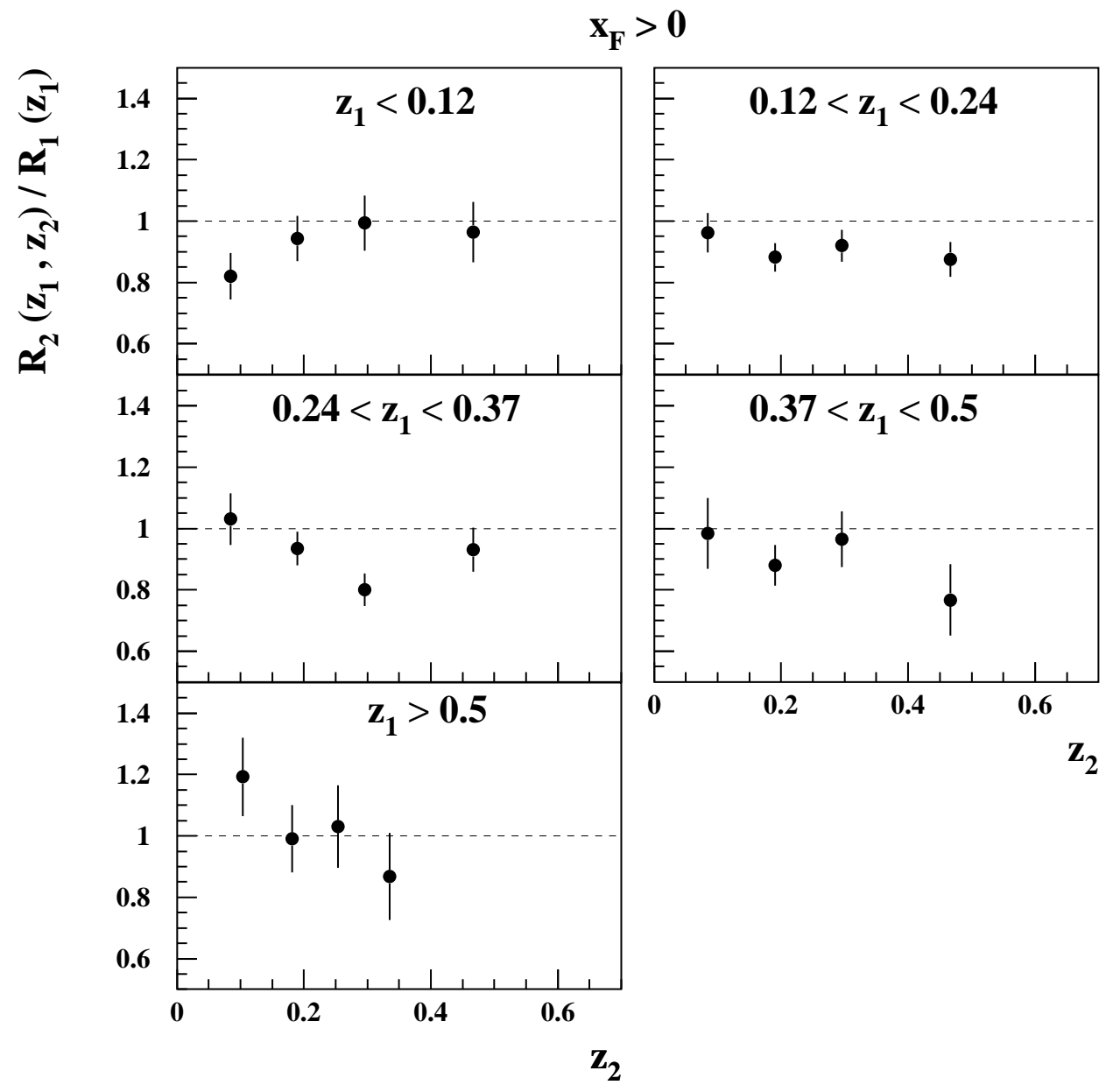

Figure 8: The double ratio $r_{21}\left(z_{1}, z_{2}\right)=R_{2}\left(z_{1}, z_{2}\right) / R_{1}\left(z_{1}\right)$ as a function of $z_{1}$ and $z_{2}$. The cut $x_{F}>0$ was applied. 


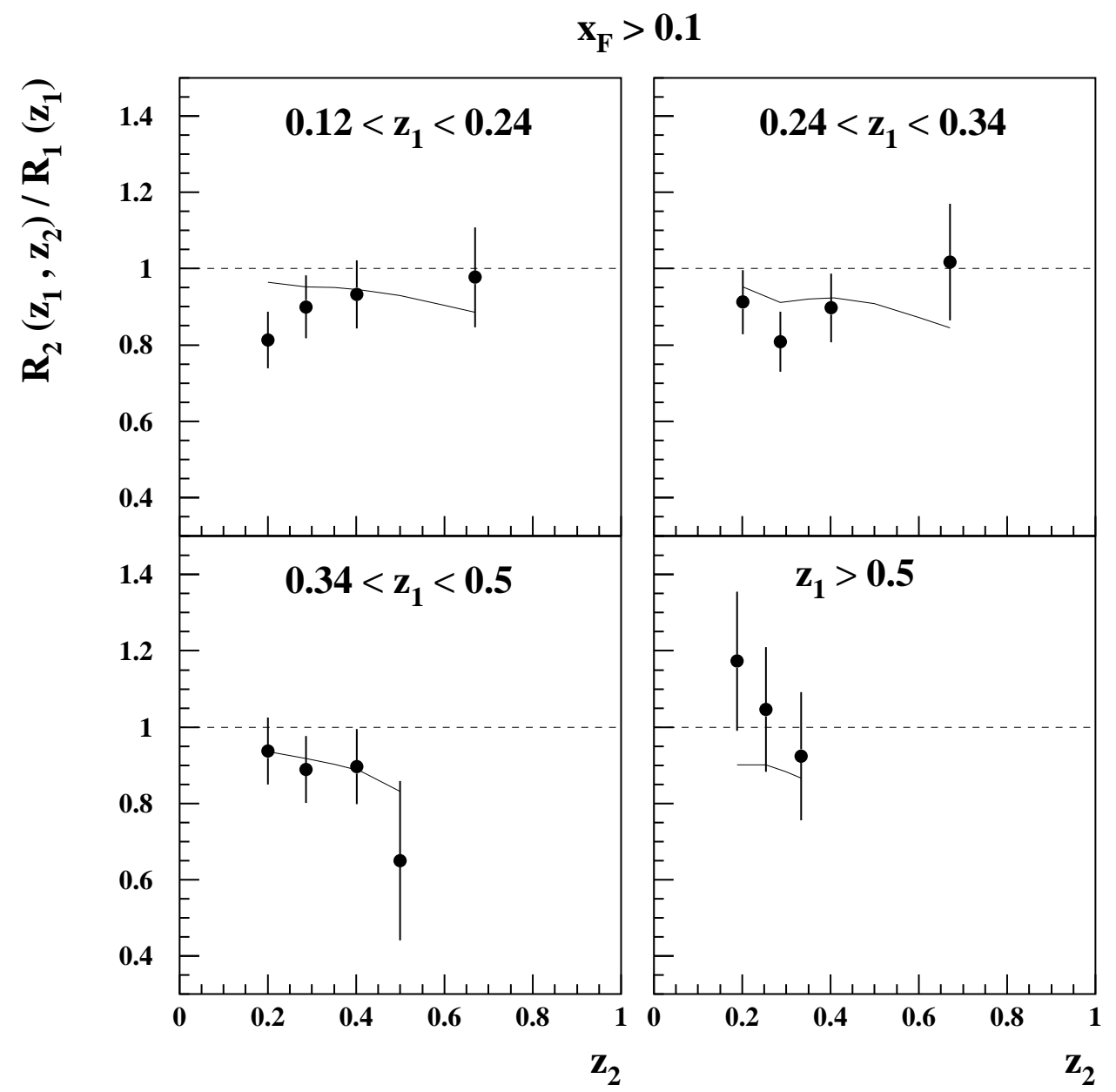

Figure 9: The same as Figure 8, but the cut $x_{F}>0.1$ is applied. The curves are predictions of the two-scale string fragmentation model. 


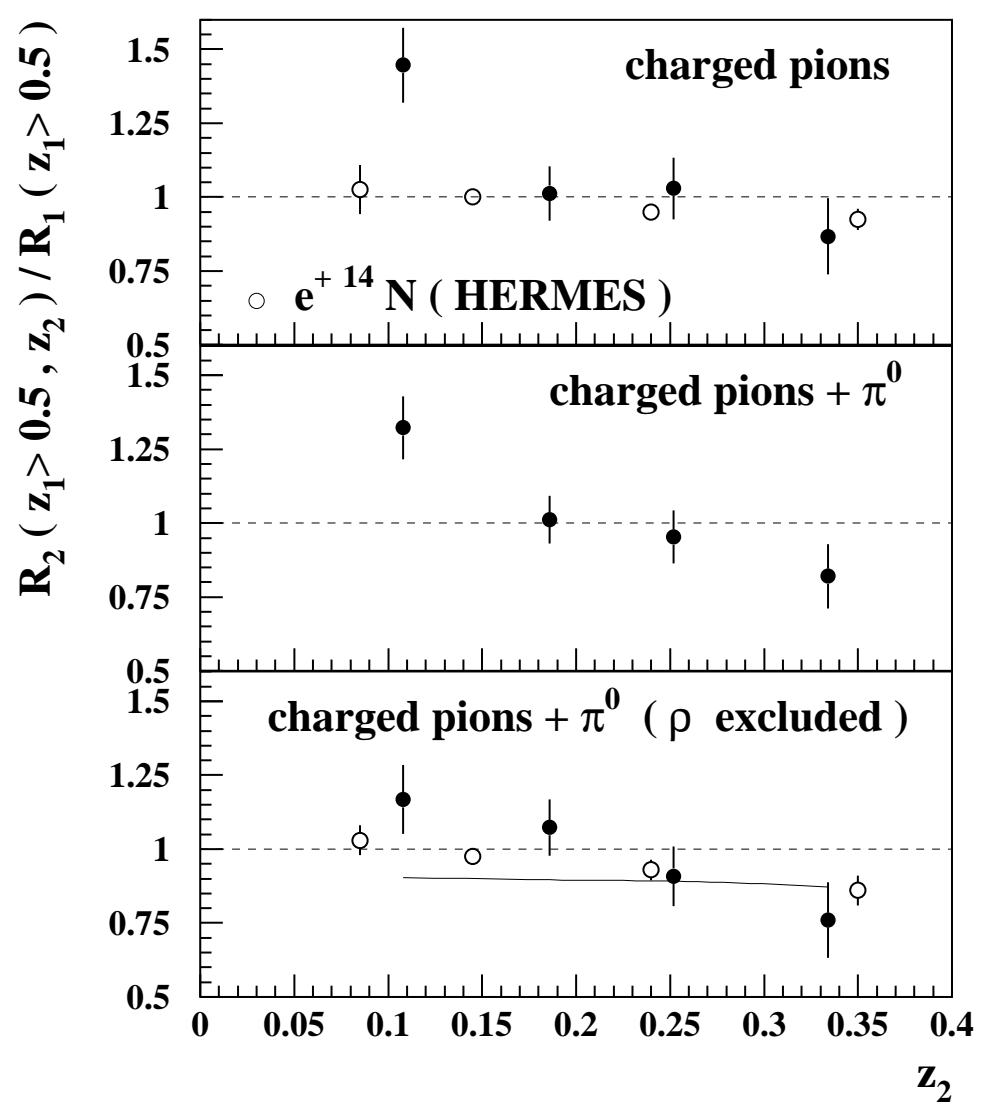

Figure 10: The double ratio $r_{21}\left(z_{1}>0.5, z_{2}\right)$ as a function of $z_{2}$ at $z_{1}>0.5$. The cut $x_{F}>0$ was applied. The curve is prediction of the two-scale string fragmentation model. 